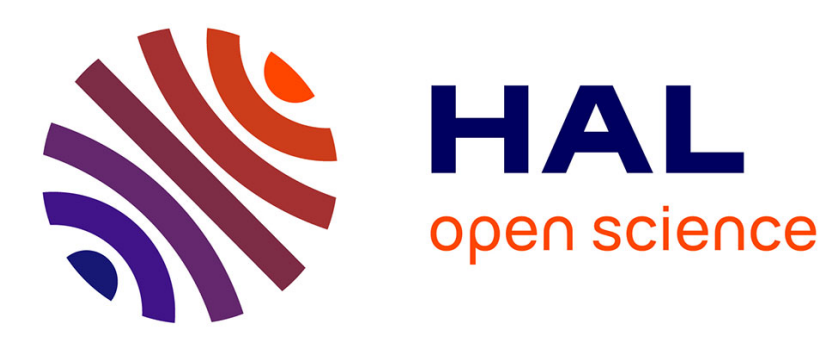

\title{
Post-transcriptional controls - adding a new layer of regulation to clock gene expression.
}

Marie Cibois, Carole Gautier-Courteille, Vincent Legagneux, Luc Paillard

\section{To cite this version:}

Marie Cibois, Carole Gautier-Courteille, Vincent Legagneux, Luc Paillard. Post-transcriptional controls - adding a new layer of regulation to clock gene expression.. Trends in Cell Biology, 2010, 20 (9), pp.533-41. 10.1016/j.tcb.2010.06.004 . inserm-00511205

\section{HAL Id: inserm-00511205 https://www.hal.inserm.fr/inserm-00511205}

Submitted on 24 Aug 2010

HAL is a multi-disciplinary open access archive for the deposit and dissemination of scientific research documents, whether they are published or not. The documents may come from teaching and research institutions in France or abroad, or from public or private research centers.
L'archive ouverte pluridisciplinaire HAL, est destinée au dépôt et à la diffusion de documents scientifiques de niveau recherche, publiés ou non, émanant des établissements d'enseignement et de recherche français ou étrangers, des laboratoires publics ou privés. 
1 Posttranscriptional controls - adding a new layer of control to clock gene

4 Marie Cibois $^{1,2,3}$, Carole Gautier-Courteille ${ }^{1,2}$, Vincent Legagneux ${ }^{1,2}$, Luc Paillard ${ }^{1,2}$

5

6

7

8 1. Université de Rennes 1, Université Européenne de Bretagne, Institut Fédératif de

9 Recherche 140, Rennes, France

10 2. CNRS UMR6061 Institut de Génétique et Développement de Rennes, France

11 3. Present address Institut de biologie du développement de Marseille, UMR6216, CNRS-

12 Université de la Méditerranée, Case 907, 13288 Marseille Luminy Cedex 09 France.

13

14

15 Corresponding author Paillard, L (luc.paillard@univ-rennes1.fr)

16

17

18 Manuscript length: 3387 words 
Living organisms undergo biochemical, physiological and behavioural cycles with periods ranging from seconds to years. The cycles with intermediate periods rely on endogenous clocks that consist of oscillating gene expression. Our goal is to illustrate the modalities and specific functions of posttranscriptional controls of gene expression (exerted on pre-mRNAs and mRNAs) in biological clocks through two examples: the circadian clock and the vertebrate somitic segmentation clock, an embryonic clock with a period far below a day. We conclude that both uniformly and cyclically exerted posttranscriptional controls underpin the set-up of clock functions.

\section{Rhythmic gene expression in oscillators}

Living organisms are submitted to periodic oscillations of biochemical, physiological and behavioural parameters that are named biological rhythms. For a given process, the periods of the cycles range from less than one second to several years (Box 1). The biorhythms are subdivided into circadian (period approximately equal to 24 hours), ultradian and infradian (respectively shorter and longer periods, See Glossary) [1].

The present review will focus on essentially two rhythms, the ultradian rhythm that underpins vertebrate somitic segmentation and the circadian rhythm. During vertebrate embryo elongation, somites (presumptive muscles and bones) periodically bud off the nonsegmented, posterior mesoderm (presomitic mesoderm). This results in a repetitive organization all along the antero-posterior axis, which is referred to as somitic segmentation. The periodic emergence of somites relies on an autonomous 'clock' within the non-segmented mesoderm that oscillates with a period ranging from 30 minutes in zebrafish to 2 hours in mice [2].

In circadian rhythms, there also exists an internal clock that is able to free-run with a period of approximately 24 hours. This clock exists in multicellular organisms, but also in yeasts [3]. This autonomous clock is temporally 'entrained' by light-dark or temperature cycles [4-6]. In mammals, it is located in the suprachiasmatic nucleus (SCN), a group of 
hypothalamic neurons. Neuronal connections between the retina and the SCN explain the entrainment by light-dark cycles, which is evidenced among others by the resetting of the clock when light-dark cycles are shifted by some hours (in experimental conditions or following long-distance travels in humans) $[4,5]$.

The mammalian circadian clock relies on eight proteins that are cyclically expressed in the SCN (Figure 1A): Clock [7], Bmal1 (Mop3) [8], Per1, Per2 and Per3 [9], Cry1 and Cry2 [10], and Rev-Erb $\alpha$ [11]. The Clock-Bmall complex controls the expression of several genes at the transcription level, among which Period (Per1 to Per3), Cryptochrome (Cryl and Cry2), and Rev-Erb $\alpha$, through its association with E-box elements. The Per-Cry protein complexes interact with and inhibit Clock-Bmall, and Rev-Erb $\alpha$ inhibits the transcription of Bmall. These two transcriptional feedback loops are responsible for the oscillations of ClockBmal1 activity that themselves account for the circadian expression of the clock outputs (Figure 1A) [4,5]. Several additional factors that modulate the mammalian circadian clock were recently identified by RNAi or proteomic approaches $[12,13]$. The circadian and the segmentation (Box 2) clocks both are set-up by transcriptional negative-feedback loops [2,14$18]$.

In addition to transcriptional loops, the control of the degradation of the proteins encoded by the clock genes determines their amounts in both clocks [17,19-21]. Several posttranslational modifications determine the activity and the stability of clock proteins [19]. Together, they represent a second layer of gene regulation in clock functions. A third layer of gene regulation must now be considered when investigating biological rhythms (Figure 1B). This layer, collectively referred to as posttranscriptional controls, encompasses all the regulations that are exerted at the RNA level (Box 3). They are mediated by ribonucleoproteic particles that include RNA-binding proteins (RNA-BPs) and non-coding RNAs, especially microRNAs (miRNAs) [22-24]. Their contributions in essential clock functions are an emerging and important field of study. 


\section{Circadian rhythms as a paradigm for dynamic posttranscriptional controls}

The first evidence for posttranscriptional controls in circadian rhythms came from pioneering work in the fruitfly Drosophila [25]. Since, oscillating mRNA stability during the circadian cycle was also demonstrated in the mammalian core pacemaker (Figure 2). The stabilities of Per2 and Cryl mRNAs vary during the cycle in mice, and, together with oscillating transcription, this results in rhythmic expression [26,27]. Woo and colleagues found that the RNA-BPs Ptbp1 and Hnrpd are able to bind to the 3' untranslated regions of Per2 and Cryl mRNAs, respectively, and cause their rapid degradation [26,27]. Furthermore, the levels of cytoplasmic Ptbp1 and Hnrpd oscillate during the circadian clock and are correlated with target mRNA decay rates. In synchronized cultured cells, the oscillations of Per2 and Cry 1 mRNAs were affected when the levels of Ptbp1 and Hrpd were reduced by RNAi. Together, these results suggest that oscillating amounts of cytoplasmic RNA-BPs may be responsible for the oscillating stability of target mRNAs that in turn determines their oscillating expression [26,27].

Rhythmic translation is another strategy to achieve cyclic expression of clock genes in the SCN, as demonstrated for Perl mRNA (Figure 2). The RNA-BP Rbm4 is cyclically expressed in-phase with Per1. It is able to bind to Per 1 mRNA and to stimulate its translation. Hence, translational stimulation by Rbm4 synergizes with transcriptional controls to amplify the level of Per1 oscillations [28]. Interestingly, only Rbm4 protein, but not Rbm4 mRNA, is cyclically expressed, indicating that Rbm4 expression is itself controlled at a translational or posttranslational (protein degradation) level [28]. It is not known whether Rbm4 is required for circadian rhythms in whole mammalian organisms, but manipulating its level in cultured mammalian cells or in Drosophila affects circadian oscillations [28,29].

In addition to RNA-BPs, microRNAs (miRNAs) also control several mRNAs within the circadian pacemaker (Figure 2). miRNAs affect both mRNA stability and translation [22]. In animals, the interactions between miRNAs and target mRNAs are mediated by limited 
sequence conservation. A miRNA can have several mRNA targets that are difficult to identify, although considering preferential evolutionary conservation improved the capacity to predict miRNA-mRNA interactions in silico [30]. Cheng and colleagues [31] showed that the miRNAs miR-219 and miR-132 have a circadian expression in the SCN, and they identified several potential mRNA targets. Per2 protein is overexpressed upon treatment with an antisense (antagomir) oligonucleotide against miR-132, which is consistent with miR-132 downregulating the translation of Per2 mRNA. Furthermore, circadian period length and light-dependent clock resetting are altered in the absence of miR-219 and miR-132 respectively [31].

The SCN emits circadian signals to other regions of the brain, including the pineal gland. This gland synthesizes melatonin during the night and this circulating hormone relays the circadian rhythm to the peripheral organs. Arylalkylamine $\mathrm{N}$-acetyltransferase (Aanat) is cyclically expressed in the pineal gland and is the rate-limiting enzyme in melatonin synthesis. Its expression is controlled at several levels, including mRNA stability and translation (Figure 2). The 3' untranslated region of Aanat mRNA contains a destabilizing element, and three rhythmically expressed RNA-BPs (Hnrnpr, Hnrnpl, Syncrip) are able to bind to this element and may play a role in the rhythmic degradation of Aanat mRNA [32]. In addition, Aanat mRNA is translated through an IRES (internal ribosome entry site), and Syncrip is able to bind to that IRES and stimulate Aanat mRNA translation. The oscillations of Syncrip protein during circadian cycles result in in-phase oscillations of Aanat mRNA translation, and manipulating the level of Syncrip impacts melatonin production in pinealocytes [33]. It is probable that the oscillations of Hnrnpr, Hnrnpl and Syncrip are themselves controlled by circadian cues sent by the SCN, but how this is achieved is unknown (Figure 2).

In addition to brain, most mammalian organs contain autonomous clocks that are entrained by cues emitted by the master clock [34], and posttranscriptional controls might operate in these peripheral clocks too. A comprehensive microarray experiment revealed 
ultradian rhythmic expression of several genes in mouse liver [35]. This might indicate some

127 ultradian clock, but an alternative cause could be mRNA degradation. If genes are transcribed

128 following circadian rhythms and the corresponding mRNAs are degraded following a

129 circadian, out-of-phase, rhythm, the mRNA levels might oscillate with a period of 12 hours $130 \quad[35]$.

131 A function for oscillating mRNA stability in circadian rhythm has also been described

132 in plants. A microarray screening in Arabidopsis thaliana identified two mRNAs whose

133 stabilities oscillate with a period of 24 hours. Disruption of the pathway responsible for the

134 rapid degradation of these mRNAs in the afternoon alters the oscillations of these mRNAs in

135 correlation with an altered circadian rhythm at the whole-plant level, indicating a link

136 between circadian rhythms in plants and specific mRNA decay [36].

\section{Clues for the importance of posttranscriptional controls in biological rhythms}

How widespread are posttranscriptional controls of gene expression in biological

rhythms? A rough estimate is provided by identifying factors that control gene expression at

141 the posttranscriptional level and that display a rhythmic circadian expression. This is the case

142 for several miRNAs in the plant Arabidopsis thaliana [37], fly heads [38] and mouse retinas $143[39]$.

144 Several examples of oscillating RNA-BPs have also been reported, in addition to the

145 factors described in the previous section. In the green alga Chlamydomonas reinhardtii, the

146 capacity of the RNA-binding complex CHLAMY1 to bind to target mRNAs follows a

147 circadian rhythm [40]. CHLAMY1 comprises two subunits that both are RNA-BPs.

148 Experimentally manipulating the level of either of these two subunits strongly interferes with

149 the circadian rhythm, suggesting that these two proteins are at the heart of the circadian clock

150 in this species [41]. The Chlamydomonas clock is entrained by temperature cycles, and both

151 subunits of CHLAMY1 are involved in temperature integration [42]. In rats, the RNA-BP

$152 \mathrm{Mbnl}$ (Muscleblind 2) that is involved in alternative splicing of pre-mRNA has an oscillatory 
expression in the pineal gland [43]. Finally, Nocturnin, a poly(A) ribonuclease (that causes mRNA decay and translational repression by removing the poly(A) tails, see Box 3), is cyclically expressed in the retina [44]. Surprisingly, mice in which the Nocturnin gene has been inactivated display normal circadian rhythms and expression of clock genes (but altered lipid metabolism or uptake) [45]. Hence, factors that control mRNA fate and display a rhythmic expression pattern can be divided into two groups: those that directly influence the clock, and those, like Nocturnin, that represent its readouts.

An additional clue to estimate the extent of translational controls in biological rhythms is to compare the levels of cycling proteins with their corresponding mRNAs. Systematic comparison of the transcriptome and the proteome of mouse liver showed that only half of the genes that exhibit rhythmic protein expression also exhibit rhythmic mRNA expression [46]. Interestingly, circadian variations in protein isoforms were also reported by these authors, which are consistent with circadian modifications of alternative splicing [46]. The strong discrepancies between transcriptome and proteome data suggest prevalent translational and/or posttranslational (protein degradation) controls of cyclic gene expression in the circadian clock.

\section{One step forward: how are cyclic posttranscriptional controls generated?}

As seen above, cyclical posttranscriptional controls are exerted on several mRNAs and in several physiological systems. In some already discussed cases, the factors involved in RNA regulations are uniformly expressed, but their activity or subcellular localisations oscillate $[26,27,41]$. The mechanisms underlying these oscillations are unknown.

The factors controlling mRNA fate may also themselves be cyclically expressed, owing to a cyclical transcriptional regulation, as demonstrated for miR-219 (see Figure 2) [31], but also owing to posttranscriptional negative-feedback loops. In Neurospora crassa, FRQ and FRH proteins form the FFC complex, which is able to recruit the RNA exosome (a multi-subunit complex involved in mRNA degradation [47]) to frq mRNA, and to thereby 
cause its degradation. Together with the capacity of FFC to repress the transcription of frq

181 gene, this posttranscriptional negative-feedback loop achieves circadian oscillations in $N$. crassa [48]. In Arabidopsis thaliana, AtGRP7 and AtGRP8 are two RNA-BPs with a circadian expression. AtGRP7 overexpression ablates circadian expression of Atgrp7 and Atgrp 8 mRNAs [49]. Both proteins are able to bind to their own pre-mRNAs and direct their splicing pathways towards mRNA isoforms that contain a premature termination codon.

186 These isoforms are rapidly degraded by the non-sense-mediated mRNA decay (NMD) pathway (see Box 3). Consequently, AtGRP7 and AtGRP8 negatively auto-regulate and crossregulate their synthesis $[50,51]$. This mechanism very probably ensures a cyclical stability of the mRNAs encoding $A t \mathrm{GRP} 7$ and $A t \mathrm{GRP} 8$, which contributes to their circadian oscillations. In mammals, the RNA-BPs Rbm4 and Syncrip display oscillating expressions [28,33].

191 It is tempting to speculate that these oscillations result from negative auto-regulations similar to plant $A t \mathrm{GRP} 7$ and $A t \mathrm{GRP} 8$ or N. crassa FRQ. Indeed, several mammalian RNA-BPs negatively regulate their own synthesis. PTBP1 and PTBP2 regulate the splicing of their own

194 respective pre-mRNAs and promote the skipping of an exon that results in an NMD sensitive 195 transcript [52,53]. They also cross-regulate each other through this splicing event [54,55].

196 Similarly, the RNA-BP Celf2 negatively autoregulates its synthesis by inhibiting the splicing of its own pre-mRNA [56]. Whether these negative auto-regulations of RNA-BPs generate oscillations, and how these putative posttranscriptional negative-feedback loops are interconnected with the master transcriptional loop, have not been tested in mammals.

Posttranscriptional controls do not need to be cyclically exerted to play a role in biological rhythms.

Transcriptional negative-feedback loops result in successive activations and repressions of gene promoters. When transcription is shut off, mRNAs decay following exponential kinetics. If the decay of a given mRNA is sufficiently rapid (short half-life) relative to the period of transcriptional oscillations, then almost complete removal of the 
mRNA will occur before transcription resumes. This situation produces oscillations of mRNA

208 of maximum amplitude. However, if the transcription resumes before the mRNA is completely degraded, then the amplitudes of the mRNA oscillations are reduced or the oscillations are damped and, at the extreme of very stable mRNAs, completely disappear.

211 Therefore, rapid mRNA degradation is required to convert switches between active and 212 inactive transcription into oscillatory amounts of the corresponding mRNAs. One could 213 predict therefore that rapid and uniform mRNA decay is instrumental in the generation of 214 short-period (ultradian) biorhythms, and this prediction has at least been partially confirmed 215 in the case of vertebrate somitic segmentation clock.

The period of the somitic segmentation clock is comprised between 30 minutes and 2

217 hours [2]. Within one period, the amounts of several tens of mRNAs oscillate [57]. It takes no 218 more than a few minutes to have a cyclic mRNA completely degraded, indicating very short 219 half-lives. The data demonstrating the occurrence of posttranscriptional controls in somitic 220 segmentation are summarized in Table 1.

The expression pattern of Lunatic Fringe (Lfng, a modulator of Notch signalling, one of the pathways required for segmentation) has been described in mice. In situ hybridizations were made with both an exonic probe to reveal the mRNA and an intronic probe to reveal sites of active transcription. The staining patterns with these two probes were very similar, demonstrating that $L f n g$ mRNA is degraded virtually as rapidly as the $L f n g$ introns [58]. Since splicing occurs co-transcriptionally, and excised introns are very rapidly degraded, these data 227 demonstrate the remarkable instability of $L f n g$ mRNA.

Reporter genes also showed that mRNA degradation is required to achieve the dynamic expression pattern of the clock genes. In Zebrafish, a GFP reporter controlled by the Herl promoter (an oscillating component of the core clock) accumulates in the presomitic 231 mesoderm owing to its high stability, suggesting a contrario the rapid decay of the 232 endogenous mRNA [59]. In Xenopus transgenic embryos, a characteristic striped expression 233 pattern of Hairy $2 a$ and Bowline, two genes downstream of the clock, is recapitulated by 
reporter mRNAs only if they contain a destabilizing element in their $3^{\prime}$ untranslated regions

235 (3'UTR) [60,61]. Taking as evidence for rapid mRNA degradation the capacity of a 3'UTR to confer upon a reporter GFP gene a striped pattern of expression, several chick or mouse clock mRNAs can be considered as unstable (Table 1 [60]). More recently, an approach combining in ovo electroporation and an inducible promoter showed that chick Lfng mRNA is destabilized by means of its 3'UTR [62].

What happens to segmentation if the rapid degradation of the cyclic mRNAs is impaired? Computational models of the zebrafish segmentation clock predict that the oscillations of the core clock genes are sustained only if the corresponding mRNA and proteins are unstable $[18,63]$, but this was not experimentally tested at the mRNA level. In

244 Zebrafish, the 'tortuga' mutant shows an altered pattern of expression of Her 1 with impaired 245 oscillations that is consistent with mRNA stabilisation [64]. The corresponding wild-type 246 gene product may therefore be responsible for the rapid decay of Herl mRNA. This gene has not been identified. In Xenopus, the RNA-BPs Celf1 and Fxrlp regulate the stability and/or 248 the translation of bound mRNAs, and knock-down of these proteins causes segmentation 249 defects $[65,66]$. This suggests that these proteins have to bind and control a subset of mRNAs 250 for correct segmentation to occur. The mRNA encoding $\mathrm{Su}(\mathrm{H})$, that is involved in Notch 251 signalling in the segmentation clock, was identified as a target of Celf1. Specifically, a 252 functional interaction between Celf1 and $S u(H)$ mRNA is required for both the degradation of 253 this mRNA and somitic segmentation [67]. Together, these data show that uniform mRNA 254 regulation plays a key role in oscillations of the segmentation clock. Continuous posttranscriptional controls were also described in the circadian clock. The 256 expression of the microRNAs miR-192 and mi-R194 in cultured mammalian cells [68], miR257122 in mouse liver [69] or bantam in fly heads [70] apparently does not follow a circadian 258 cycle (although miR-122 is cyclically transcribed but remains at approximately constant 259 levels due to a long high-life [69]). All these miRNAs continuously downregulate identified 260 target mRNAs encoding proteins involved in the circadian clock, and manipulating their 
levels modifies the period and/or amplitude of circadian oscillations [68-70]. Other examples

262 are given by Perl and Per3 mRNAs that are uniformly unstable in NIH3T3 cells and 263 transgenic mice, respectively [71,72]. The circadian oscillations of Per3 mRNA are strongly 264 modified when its mRNA degradation element is deleted [71]. Hence, constant 265 posttranscriptional repression may be required in some instances to achieve optimal circadian 266 oscillations in addition to cyclical posttranscriptional controls of gene expression.

\section{Concluding remarks and future directions}

The comparison of the segmentation and circadian clocks paves the way for future researches (Box 4). Both mRNA degradation and translation, mediated by RNA-BPs and miRNAs, have recognized functions in the circadian clock. In several instances, translational efficiency and mRNA degradation oscillate in the circadian clock, and these oscillations fully contribute to the clock. By contrast, the only known mode of posttranscriptional control in the segmentation clock is constant mRNA degradation. In fact, we might simply lack data concerning the different modes of posttranscriptional controls in the segmentation clock. Using the circadian clock as a paradigm for posttranscriptional controls in clocks, we recommend that the various modes of oscillating posttranscriptional controls should be carefully investigated in the segmentation clock. Furthermore, most but not all known modes of posttranscriptional controls were described in the circadian clock. Specifically, we know nothing about the subcellular localization and the putative localized translation of the mRNAs encoding factors of the clock. It might be of interest to investigate these points in the 282 regulation of mammalian circadian clock considering their recognized importance in neurons [73].

Another question is whether there exist human diseases caused by posttranscriptional defects in clocks. Congenital vertebral malformations are often of genetic origin. Some of them were associated with mutations affecting genes of the segmentation clock, but the aetiology of most of them is unknown [74]. Factors involved in posttranscriptional regulations 
in somitic segmentation, most of which were not identified, will be potential candidates for

289 causing these syndromes. Also several human troubles arise from defects in the circadian 290 clock, such as sleep disorders. Interestingly, fragile X patients suffer from sleep disorders 291 [75]. This syndrome is a consequence of impaired expression of the RNA-BP FMR1, and 292 Fmrl KO mice display an altered circadian rhythm [76]. Fragile X syndrome provides 293 therefore a link between posttranscriptional controls, human pathology and the circadian 294 clock, and it can be anticipated that this will not remain an isolated example.

A last issue is the extent of posttranscriptional controls in clocks. Several inactivations 296 of gene encoding RNA-BPs were reported in mice. Some of them may be at the origin of 297 circadian troubles that remained unnoticed up to now, and this would merit careful 298 reinvestigation. For the RNA-BPs whose inactivations lead to clock troubles, the arising 299 question will be the identity of the mRNAs that are normally associated with that protein and 300 are deregulated upon its inactivation (and whose deregulation is responsible of the observed 301 troubles). Recent technological breakthroughs allow some optimism concerning our capacity 302 to ask that question. "CLIP" (Cross-linking and immunoprecipitation) allows the co303 immunoprecipitation of RNA-BPs and associated RNAs [77]. Combined with next-generation 304 sequencing, it permits the genome-wide identification of the RNAs bound by a protein 305 ('CLIPseq') [78-80]. Maps of the interactions between miRNAs and mRNAs were drawn 306 from Argonaute CLIPseq [81,82]. Together, these recent technologies will provide us with a 307 genome-wide characterization of the network of posttranscriptional controls in virtually any 308 cell type, including those subject to clock oscillations, and will allow us fully appreciating the 309 extent of posttranscriptional controls in clocks.

\section{Acknowledgments}

We thank S. Hardy and H.B. Osborne for critical evaluation of the manuscript. V.L. is

313 a staff member of the INSERM (Institut National de la Santé et de la Recherche Médicale). 
Work in the authors' laboratory is supported by a grant from the Agence Nationale de la

\section{References}

3181 Schibler, U. and Naef, F. (2005) Cellular oscillators: rhythmic gene expression and 319 metabolism. Curr Opin Cell Biol 17 (2), 223-229

3202 Pourquie, O. (2003) The segmentation clock: converting embryonic time into spatial

3 Eelderink-Chen, Z. et al. (2010) A circadian clock in Saccharomyces cerevisiae. Proc Natl Acad Sci U S A 107 (5), 2043-2047

4 Takahashi, J.S. et al. (2008) The genetics of mammalian circadian order and disorder: implications for physiology and disease. Nat Rev Genet 9 (10), 764-775

5 Gery, S. and Koeffler, H.P. (2010) Circadian rhythms and cancer. Cell Cycle 9 (6)

6 Rensing, L. and Ruoff, P. (2002) Temperature effect on entrainment, phase shifting, and amplitude of circadian clocks and its molecular bases. Chronobiol Int 19 (5), 807864

7 Vitaterna, M.H. et al. (1994) Mutagenesis and mapping of a mouse gene, Clock, essential for circadian behavior. Science 264 (5159), 719-725

8 Bunger, M.K. et al. (2000) Mop3 is an essential component of the master circadian pacemaker in mammals. Cell 103 (7), 1009-1017

9 Zheng, B. et al. (2001) Nonredundant roles of the mPer1 and mPer2 genes in the mammalian circadian clock. Cell 105 (5), 683-694

10 Kume, K. et al. (1999) mCRY1 and mCRY2 are essential components of the negative limb of the circadian clock feedback loop. Cell 98 (2), 193-205

11 Preitner, N. et al. (2002) The orphan nuclear receptor REV-ERBalpha controls circadian transcription within the positive limb of the mammalian circadian oscillator. Cell 110 (2), 251-260

12 Zhang, E.E. et al. (2009) A genome-wide RNAi screen for modifiers of the circadian clock in human cells. Cell 139 (1), 199-210

13 Robles, M.S. et al. (2010) Identification of RACK1 and protein kinase Calpha as integral components of the mammalian circadian clock. Science 327 (5964), 463-466

14 Hardin, P.E. et al. (1990) Feedback of the Drosophila period gene product on circadian cycling of its messenger RNA levels. Nature 343 (6258), 536-540

15 Dunlap, J.C. and Loros, J.J. (2006) How fungi keep time: circadian system in Neurospora and other fungi. Curr Opin Microbiol 9 (6), 579-587

16 Hardin, P.E. (2005) The circadian timekeeping system of Drosophila. Curr Biol 15 (17), R714-722

17 Ko, C.H. and Takahashi, J.S. (2006) Molecular components of the mammalian circadian clock. Hum Mol Genet 15 Spec No 2, R271-277

18 Giudicelli, F. et al. (2007) Setting the tempo in development: an investigation of the zebrafish somite clock mechanism. PLoS Biol 5 (6), e150

19 Mehra, A. et al. (2009) Post-translational modifications in circadian rhythms. Trends Biochem Sci 34 (10), 483-490

20 Lee, H. et al. (2009) Essential roles of CKIdelta and CKIepsilon in the mammalian circadian clock. Proc Natl Acad Sci U S A 106 (50), 21359-21364

21 Hirata, H. et al. (2004) Instability of Hes7 protein is crucial for the somite segmentation clock. Nat Genet 36 (7), 750-754 
22 Filipowicz, W. et al. (2008) Mechanisms of post-transcriptional regulation by microRNAs: are the answers in sight? Nat Rev Genet 9 (2), 102-114

23 Glisovic, T. et al. (2008) RNA-binding proteins and post-transcriptional gene regulation. FEBS Lett 582 (14), 1977-1986

24 Keene, J.D. (2010) Minireview: global regulation and dynamics of ribonucleic Acid. Endocrinology 151 (4), 1391-1397

So, W.V. and Rosbash, M. (1997) Post-transcriptional regulation contributes to Drosophila clock gene mRNA cycling. Embo J 16 (23), 7146-7155 Woo, K.C. et al. (2009) Mouse period 2 mRNA circadian oscillation is modulated by PTB-mediated rhythmic mRNA degradation. Nucleic Acids Res 37 (1), 26-37

oo, K.C. et al. (2010) Circadian amplitude of cryptochrome 1 is modulated by mRNA stability regulation via cytoplasmic hnRNP D oscillation. Mol Cell Biol 30 (1), 197-205

28 Kojima, S. et al. (2007) LARK activates posttranscriptional expression of an essential mammalian clock protein, PERIOD1. Proc Natl Acad Sci U S A 104 (6), 1859-1864

29 Huang, Y. et al. (2009) Altered LARK expression perturbs development and physiology of the Drosophila PDF clock neurons. Mol Cell Neurosci 41 (2), 196-205

30 Bartel, D.P. (2009) MicroRNAs: target recognition and regulatory functions. Cell 136 (2), 215-233

31 Cheng, H.Y. et al. (2007) microRNA modulation of circadian-clock period and entrainment. Neuron 54 (5), 813-829

32 Kim, T.D. et al. (2005) Rhythmic serotonin N-acetyltransferase mRNA degradation is essential for the maintenance of its circadian oscillation. Mol Cell Biol 25 (8), 32323246

33 Kim, T.D. et al. (2007) Rhythmic control of AANAT translation by hnRNP Q in circadian melatonin production. Genes Dev 21 (7), 797-810

34 Kornmann, B. et al. (2007) System-driven and oscillator-dependent circadian transcription in mice with a conditionally active liver clock. PLoS Biol 5 (2), e34

35 Hughes, M.E. et al. (2009) Harmonics of circadian gene transcription in mammals. PLoS Genet 5 (4), e1000442

36 Lidder, P. et al. (2005) Circadian control of messenger RNA stability. Association with a sequence-specific messenger RNA decay pathway. Plant Physiol 138 (4), 23742385

37 Sire, C. et al. (2009) Diurnal oscillation in the accumulation of Arabidopsis microRNAs, miR167, miR168, miR171 and miR398. FEBS Lett 583 (6), 1039-1044

38 Yang, M. et al. (2008) Circadian regulation of a limited set of conserved microRNAs in Drosophila. BMC Genomics 9, 83

$39 \mathrm{Xu}$, S. et al. (2007) MicroRNA (miRNA) transcriptome of mouse retina and identification of a sensory organ-specific miRNA cluster. J Biol Chem 282 (34), 25053-25066

40 Mittag, M. et al. (1994) Circadian expression of the luciferin-binding protein correlates with the binding of a protein to the 3' untranslated region of its mRNA. Proc Natl Acad Sci U S A 91 (12), 5257-5261

41 Iliev, D. et al. (2006) A heteromeric RNA-binding protein is involved in maintaining acrophase and period of the circadian clock. Plant Physiol 142 (2), 797-806

42 Voytsekh, O. et al. (2008) Both subunits of the circadian RNA-binding protein CHLAMY1 can integrate temperature information. Plant Physiol 147 (4), 2179-2193

43 Kim, J.S. et al. (2009) Muscleblind-like 2: circadian expression in the mammalian pineal gland is controlled by an adrenergic-cAMP mechanism. J Neurochem 110 (2), 756-764

44 Baggs, J. and Green, C. (2003) Nocturnin, a Deadenylase in Xenopus laevis Retina. A Mechanism for Posttranscriptional Control of Circadian-Related mRNA. Curr Biol 13 (3), 189-198 
45 Green, C.B. et al. (2007) Loss of Nocturnin, a circadian deadenylase, confers resistance to hepatic steatosis and diet-induced obesity. Proc Natl Acad Sci U S A 104 (23), 9888-9893

46 Reddy, A.B. et al. (2006) Circadian orchestration of the hepatic proteome. Curr Biol $16(11), 1107-1115$

47 Houseley, J. et al. (2006) RNA-quality control by the exosome. Nat Rev Mol Cell Biol 7 (7), 529-539 Guo, J. et al. (2009) The exosome regulates circadian gene
posttranscriptional negative feedback loop. Cell 138 (6), 1236-1246

49 Heintzen, C. et al. (1997) AtGRP7, a nuclear RNA-binding protein as a component of a circadian-regulated negative feedback loop in Arabidopsis thaliana. Proc Natl Acad Sci U S A 94 (16), 8515-8520

50 Schoning, J.C. et al. (2007) Auto-regulation of the circadian slave oscillator component AtGRP7 and regulation of its targets is impaired by a single RNA recognition motif point mutation. Plant J 52 (6), 1119-1130

51 Schoning, J.C. et al. (2008) Reciprocal regulation of glycine-rich RNA-binding proteins via an interlocked feedback loop coupling alternative splicing to nonsensemediated decay in Arabidopsis. Nucleic Acids Res 36 (22), 6977-6987

52 Wollerton, M.C. et al. (2004) Autoregulation of polypyrimidine tract binding protein by alternative splicing leading to nonsense-mediated decay. Mol Cell 13 (1), 91-100

53 Rahman, L. et al. (2002) Alternative splicing of brain-specific PTB defines a tissuespecific isoform pattern that predicts distinct functional roles. Genomics 80 (3), 245249

54 Spellman, R. et al. (2007) Crossregulation and functional redundancy between the splicing regulator PTB and its paralogs nPTB and ROD1. Mol Cell 27 (3), 420-434

55 Boutz, P.L. et al. (2007) A post-transcriptional regulatory switch in polypyrimidine tract-binding proteins reprograms alternative splicing in developing neurons. Genes Dev 21 (13), 1636-1652

56 Dembowski, J.A. and Grabowski, P.J. (2009) The CUGBP2 splicing factor regulates an ensemble of branchpoints from perimeter binding sites with implications for autoregulation. PLoS Genet 5 (8), e1000595

57 Dequeant, M.L. et al. (2006) A complex oscillating network of signaling genes underlies the mouse segmentation clock. Science 314 (5805), 1595-1598

58 Morales, A. et al. (2002) Periodic Lunatic fringe expression is controlled during segmentation by a cyclic transcriptional enhancer responsive to notch signaling. Dev Cell 3, 63-74

59 Gajewski, M. et al. (2003) Anterior and posterior waves of cyclic her1 gene expression are differentially regulated in the presomitic mesoderm of zebrafish. Development 130 (18), 4269-4278

60 Davis, R. et al. (2001) Molecular targets of vertebrate segmentation: two mechanisms control segmental expression of Xenopus Hairy2 during somite formation. Dev Cell 1, 553-565

61 Hitachi, K. et al. (2008) Tbx6, Thylacine1, and E47 synergistically activate bowline expression in Xenopus somitogenesis. Dev Biol 313 (2), 816-828

62 Hilgers, V. et al. (2005) In vivo analysis of mRNA stability using the Tet-Off system in the chicken embryo. Dev Biol 284 (2), 292-300

63 Lewis, J. (2003) Autoinhibition with transcriptional delay: a simple mechanism for the zebrafish somitogenesis oscillator. Curr Biol 13 (16), 1398-1408

64 Dill, K.K. and Amacher, S.L. (2005) tortuga refines Notch pathway gene expression in the zebrafish presomitic mesoderm at the post-transcriptional level. Dev Biol 287 (2), 225-236 
Gautier-Courteille, C. et al. (2004) EDEN-BP-dependent post-transcriptional regulation of gene expression in Xenopus somitic segmentation. Development 131 (24), 6107-6117

66 Huot, M.E. et al. (2005) The RNA-binding protein fragile X-related 1 regulates somite formation in Xenopus laevis. Mol Biol Cell 16 (9), 4350-4361

Cibois, M. et al. (2010) A strategy to analyze the phenotypic consequences of inhibiting

68 Nagel, R. et al. (2009) The miRNA-192/194 cluster regulates the Period gene family and the circadian clock. Febs $J 276$ (19), 5447-5455

69 Gatfield, D. et al. (2009) Integration of microRNA miR-122 in hepatic circadian gene expression. Genes Dev 23 (11), 1313-1326

70 Kadener, S. et al. (2009) A role for microRNAs in the Drosophila circadian clock. Genes Dev 23 (18), 2179-2191

71 Kwak, E. et al. (2006) Essential role of 3'-untranslated region-mediated mRNA decay in circadian oscillations of mouse Period3 mRNA. J Biol Chem 281 (28), 1910019106

72 Wilsbacher, L.D. et al. (2002) Photic and circadian expression of luciferase in mPeriod1-luc transgenic mice invivo. Proc Natl Acad Sci US A 99 (1), 489-494

73 Wang, D.O. et al. (2010) Spatially restricting gene expression by local translation at synapses. Trends Neurosci 33 (4), 173-182

74 Giampietro, P.F. et al. (2009) Progress in the understanding of the genetic etiology of vertebral segmentation disorders in humans. Ann N Y Acad Sci 1151, 38-67

75 Gould, E.L. et al. (2000) Melatonin profiles and sleep characteristics in boys with fragile X syndrome: a preliminary study. Am J Med Genet 95 (4), 307-315

76 Zhang, J. et al. (2008) Fragile X-related proteins regulate mammalian circadian behavioral rhythms. Am J Hum Genet 83 (1), 43-52

77 Ule, J. et al. (2003) CLIP identifies Nova-regulated RNA networks in the brain. Science 302 (5648), 1212-1215

78 Licatalosi, D.D. et al. (2008) HITS-CLIP yields genome-wide insights into brain alternative RNA processing. Nature 456 (7221), 464-469

79 Sanford, J.R. et al. (2009) Splicing factor SFRS1 recognizes a functionally diverse landscape of RNA transcripts. Genome Res 19 (3), 381-394

80 Yeo, G.W. et al. (2009) An RNA code for the FOX2 splicing regulator revealed by mapping RNA-protein interactions in stem cells. Nat Struct Mol Biol 16 (2), 130-137

81 Chi, S.W. et al. (2009) Argonaute HITS-CLIP decodes microRNA-mRNA interaction maps. Nature 460 (7254), 479-486

82 Zisoulis, D.G. et al. (2010) Comprehensive discovery of endogenous Argonaute binding sites in Caenorhabditis elegans. Nat Struct Mol Biol 17 (2), 173-179

83 Robertson, J.B. et al. (2008) Real-time luminescence monitoring of cell-cycle and respiratory oscillations in yeast. Proc Natl Acad Sci U S A 105 (46), 17988-17993

84 Veldhuis, J.D. et al. (1986) Spectrum of the pulsatile characteristics of LH release in normal men. $J$ Androl 7 (2), 83-92

85 Knight, J.E. et al. (2000) mRNA stability and polysome loss in hibernating Arctic ground squirrels (Spermophilus parryii). Mol Cell Biol 20 (17), 6374-6379.

86 Hoppensteadt, F.C. and Keller, J.B. (1976) Synchronization of periodical cicada emergences. Science 194 (4262), 335-337

87 Kawamura, A. et al. (2005) Zebrafish hairy/enhancer of split protein links FGF signaling to cyclic gene expression in the periodic segmentation of somites. Genes Dev 19 (10), 1156-1161

88 Kuersten, S. and Goodwin, E.B. (2003) The power of the 3' UTR: translational control and development. Nat Rev Genet 4 (8), 626-637 
51789 Mauxion, F. et al. (2009) BTG/TOB factors impact deadenylases. Trends Biochem Sci $518 \quad 34(12), 640-647$

51990 Garceau, N.Y. et al. (1997) Alternative initiation of translation and time-specific phosphorylation yield multiple forms of the essential clock protein FREQUENCY. Cell 89 (3), 469-476

91 Le Hir, H. and Seraphin, B. (2008) EJCs at the heart of translational control. Cell 133 (2), 213-216

92 Kamath, R. et al. (2003) Systematic functional analysis of the Caenorhabditis elegans genome using RNAi. Nature 421, 231-237

93 Dietzl, G. et al. (2007) A genome-wide transgenic RNAi library for conditional gene inactivation in Drosophila. Nature 448 (7150), 151-156 


\section{Glossary box}

3' Untranslated Region (3'UTR): Region of the mRNA 3' to the translation stop codon.

530 Alternative splicing: Various ways to skip introns and splice exons. This mechanism generates a large diversity of mRNA molecules from a single gene. Alternative splicing includes mutually exclusive exons (where splicing leads to the inclusion of either of two exons), exon skipping, intron retention, alternative 5' or 3' splice sites (leading to the retention

534 of all or only part of an exon) and alternative terminal exons.

535 Circadian rhythm: A cycle one day long (Latin circa, about, and dies, day). The period of a 536 circadian rhythm is $24 \mathrm{~h}$ when the organism is grown under a light-dark cycle $(12 \mathrm{~h} \mathrm{light,} 12 \mathrm{~h}$ darkness), and about $24 \mathrm{~h}$ when the organism is released into free-running condition. Several parameters cycle in circadian rhythms, the most obvious one in mammals being sleep and 539 wake.

540 Free-running rhythm: Circadian rhythm in the absence of external cues (like constant 541 darkness and temperature).

542 Half-life. Time required in the absence of synthesis to achieve degradation of half the initial 543 amount of a molecule (like an mRNA).

544 Infradian rhythm: A cycle of length above $24 \mathrm{~h}$.

545 Melatonin: Circulating hormone secreted by the pineal gland during the night in mammals. It 546 relays the circadian rhythm imposed by the central nervous system to the peripheral organs. miRNA (micro RNA): Short double-stranded RNA, encoded by the genome, that controls gene expression at several levels. In vertebrates, a prevalent feature of miRNAs is their 549 capacity to specifically repress the translation of target mRNAs by (limited) sequence 550 complementarity.

551 Period: Time interval between two reference points (two peaks for example). Inverse of 552 frequency.

553 Presomitic mesoderm: Posterior, non-segmented mesoderm, in which the segmentation 554 clock is active and from which segmented somites periodically bud off. 
555 Somites: Transient embryonic repeated mesodermal structures. They are the origin of adult 556 skeletal muscles, bones and derm.

557 Somitic segmentation: Organisation of the somites as repeated units along the embryonic 558 antero-posterior axis.

559 Suprachiasmatic nucleus (SCN): A region of the hypothalamus. The master circadian clock 560 is located within the SCN.

561 Ultradian rhythm: A cycle of length shorter than 24h (e.g. the segmentation clock). 


\section{Box 1. Some examples of biological rhythms}

563

564 Depending on the period, biorhythms are classified as ultradian (period $\mathrm{T}<24 \mathrm{~h}$ ), infradian

$565(\mathrm{~T}>24 \mathrm{~h})$ and circadian $(\mathrm{T} \sim 24 \mathrm{~h})$. Ultradian rhythms include heart beating $(\mathrm{T}=$ fractions of

566 seconds to seconds), sleep episodes ( $\mathrm{T}=$ tens of minutes), respiratory oscillations in yeasts

$567(\mathrm{~T}=1-5 \mathrm{~h}[83])$, somitic segmentation in vertebrates $(\mathrm{T}=30$ minutes in Zebrafish, $2 \mathrm{~h}$ in mice

568 [2]), or pulses of LH secretion by the pituitary gland (T 3h in men [84]). Infradian rhythms

569 include successions of torpor and arousal during the hibernation of small mammals

570 ( $\mathrm{T}=$ several days [85]), female menstrual cycles $(\mathrm{T}=$ several days to several months), annual

571 rhythms (flowering of most plants), and even pluri-annual rhythms such as the emergence of

572 Cicada [86]. 


\section{Box 2. The vertebrate segmentation clock.}

574 Please refer to the accompanying figure.

575 Title of the figure "The zebrafish core segmentation clock"

576

577 In zebrafish, the core segmentation clock consists of Her1 and Her7 proteins (see Figure).

578 Homodimers or heterodimers of these proteins bind to their own promoters and repress their 579 transcription. Taking into account transcriptional and translational delays, this results in 580 oscillating levels of these proteins. Furthermore, Her1/7 duplexes repress the transcription of 581 Delta-C, a transmembrane Notch ligand. When bound by its ligand, the Notch transmembrane 582 receptor undergoes a limited proteolysis that releases the Notch intracellular domain (NICD) 583 in the cytoplasm. NICD is then translocated to the nucleus. Together with $\mathrm{Su}(\mathrm{H})$ protein, the 584 NICD stimulates the transcription of target genes including Herl and Her7. The stimulation 585 of Her $1 / 7$ transcription by Delta-C expressed in adjacent cells, and the ensuing repression of 586 Delta- $C$ gene by Her1/7 achieves coordinated oscillations in neighbouring cells $[18,63]$. 587 Her13.2 reinforces the transcriptional inhibition mediated by Her1/7, and it is controlled by 588 the FGF pathway. This links the Notch and FGF signalling pathways [87]. Several other 589 genes are downstream of Her1/7 and are involved in somitic segmentation. In amniotes 590 (chick, mouse), the segmentation clock is more complex. It requires oscillations of the Notch 591 modulator Lunatic fringe, and of tens of mRNAs that encode proteins belonging to the FGF 592 and Wnt signalling pathways in addition to Notch [57]. 
595 Please refer to the accompanying figure.

596 Title of the figure "pre-mRNA and mRNA fate in eukaryotic cells"

The posttranscriptional controls are exerted on RNA molecules and are indicated in red on the

599 figure. Concomitantly with nuclear transcription, pre-mRNAs are matured to mRNAs. Pre600 mRNA maturation refers to three events: 5' capping, 3' cleavage and polyadenylation, and 601 intron excision coupled with exon splicing. Most pre-mRNAs can be cleaved and polyadenylated at several sites (alternative cleavage/polyadenylation) and/or undergo several splicing patterns (alternative splicing. In the figure, the second exon is either skipped or 604 spliced). Due to alternative cleavage/polyadenylation and alternative splicing, a large variety 605 of mRNAs can be obtained from a given pre-mRNA.

606 After nucleo-cytoplasmic export, mRNA translation and decay are controlled, and the 3' 607 poly(A) tail is a major site for these controls. Polyadenylated mRNAs are much more actively 608 translated than deadenylated mRNAs. The initiation factor eIF4G, that recruits the small 609 ribosomal subunit, is able to interact simultaneously with the 5' cap-binding protein eIF4E and 610 the 3' Poly(A) binding protein. The connection between mRNA 5' (cap) and 3' (poly(A) tail) 611 ends strongly stimulates translation [88]. In addition, polyadenylated mRNAs are much more 612 stable than deadenylated mRNAs. For most mRNAs, deadenylation is the rate-limiting step of 613 mRNA decay, and several factors that control mRNA stability do so by regulating the 614 deadenylation rate. In higher eukaryotes, the major pathway for mRNA decay is poly(A) tail 615 removal (deadenylation) followed by RNA exosome-mediated $3^{\prime}$ to $5^{\prime}$ exonucleolytic 616 degradation. [89]. The 5'-most AUG codon is generally the translation initiation codon, but 617 more distal initiation codons can also be used (alternative initiation of translation), resulting in 618 alternative protein isoforms. This mechanism was described for instance for the mRNA that 619 encodes FRQ, a component of the N. Crassa circadian clock [90]. 
620 Nuclear and cytoplasmic controls are tightly coupled. A complex (EJC, exon junction 621 complex) is assembled during splicing immediately upstream of exon junctions, and remains 622 associated with the mRNA during nucleocytoplasmic export. This hallmark of a nuclear event 623 then influences cytoplasmic mRNA translation and degradation [91]. For example, the EJC is 624 involved in the recognition and rapid degradation of mRNAs containing a premature stop 625 codon by the 'nonsense-mediated mRNA decay' (NMD) pathway [91]. In addition, 626 alternative splicing can lead to mature transcripts that contain alternative $3^{\prime}$ untranslated 627 regions (3'UTR), that are instrumental in mRNA stability and translation [88]. Consequently, 628 alternative cleavage/polyadenylation or splicing impacts mRNA half-life or translation. 


\section{Box 4. Future questions}

630 - Uniform mRNA instability is the only mode of posttranscriptional controls demonstrated in

631 the segmentation clock. Do oscillating mRNA stability and/or oscillating mRNA translation 632 also play a role?

633 - In the circadian clock, the described mechanisms relate to most posttranscriptional controls

634 found to be governing the expression of other non-clock-related gene programs, but mRNA 635 intracellular traffic and local translation were not reported. Since they are prevalent 636 mechanisms in neurons [73], one could ask if they have a function in the circadian clock.

637 - A posttranscriptional feedback loop was demonstrated in N. crassa circadian clock [48], and 638 the levels of some RNA-BPs oscillate in mammalian circadian clocks [28,33]. Are there 639 posttranscriptional feedback loops in vertebrate clocks that could account for the oscillations 640 of these RNA-BPs?

641 - Systematic gene inactivations were reported in lower metazoans [92,93], and several genes 642 were disrupted by homologous recombination in mice. Some of them encode RNA-BPs or 643 miRNAs. Which inactivations lead to clock troubles, demonstrating an involvement of the 644 corresponding gene products in clock setting or robustness?

645 - What are the posttranscriptional networks in clocks? For the RNA-BPs and the miRNAs that 646 are involved in clocks, what are the associated mRNAs?

647 - Are deregulations of posttranscriptional networks in clocks at the origin of human diseases? 


\section{Figure 1. The mammalian circadian clock and its three layers of control}

650 (a) Master circadian pacemaker in the suprachiasmatic nucleus (SCN). The Clock-Bmal1 651 complex directly stimulates the transcription of Per, Cry, Rev-Erb $\alpha$, and of output clock652 controlled genes (CCGs) via binding to the E-box. Oscillatory activity of the Clock-Bmall 653 complex is achieved by two negative feedback loops: the Per-Cry complex inhibits Clock654 Bmal1, and Bmall transcription is repressed by binding of Rev-Erbo to the RRE (ROR 655 response element). (b) Relationships between transcriptional, posttranscriptional and 656 posttranslational layers in the control of $\mathrm{Per}$ genes expression. Since Per proteins contribute to 657 the control of the Clock-Bmall complex, fine-tuning their levels is required to obtain 658 oscillations of clock genes. The levels of Per proteins are regulated at a transcriptional level 659 (yellow layer) by the Clock-Bmall complex (see Figure 1a). They are regulated at a 660 posttranslational level too (green layer), among others as Casein-kinase $1-\delta$ and $-\varepsilon$ mediate Per 661 phosphorylation that targets them to ubiquitin/proteasome degradation $[19,20]$. Recent results 662 demonstrate that a third layer (posttranscriptional controls, red) should be added to complete 663 the picture. The oscillating controls (transcription, mRNA translation and degradation) are in 664 capital letters.

666 Figure 2. Posttranscriptional controls exerted on mRNAs encoding proteins involved in 667 circadian rhythms

668 Arrows and blunt-end lines towards ribosomes (brown) indicate stimulation and inhibition, respectively, of mRNA translation. Arrows towards the exonucleolytic enzyme (yellow)

670 indicate stimulation of mRNA decay. The sinusoidal symbols on the right of the factors 671 involved in posttranscriptional controls indicate oscillating levels of these factors. (a) 672 Components of the master circadian clock in the SCN. (b) Aanat, a pineal, rate-limiting enzyme in melatonin synthesis. 


\begin{tabular}{|c|c|c|c|}
\hline Gene & Function in the clock & $\begin{array}{l}\text { Evidence for posttranscriptional } \\
\text { controls }\end{array}$ & References \\
\hline $\begin{array}{l}\text { Llnfg in } \\
\text { amniotes }\end{array}$ & $\begin{array}{l}\text { Encodes modulator of Notch } \\
\text { signalling }\end{array}$ & $\begin{array}{l}\text { mRNA instability inferred from } \\
\text { expression pattern in mice; } 3 \text { 'UTR of } \\
\text { chick mRNA confers rapid degradation to } \\
\text { a reporter mRNA }\end{array}$ & {$[58,62]$} \\
\hline zebrafish Herl & $\begin{array}{l}\text { Encodes component of the core } \\
\text { clock }\end{array}$ & $\begin{array}{l}\text { The expression pattern of a reporter } \\
\text { mRNA controlled by Her } 1 \text { promoter is } \\
\text { different from that of endogenous Herl } \\
\text { due to increased mRNA stability. } \\
\text { Expression pattern in the Tortuga mutant } \\
\text { consistent with Tortuga gene product } \\
\text { being responsible for Her } 1 \text { mRNA } \\
\text { instability }\end{array}$ & {$[59,64]$} \\
\hline $\begin{array}{l}\text { Xenopus Hairy } \\
2 a, \text { Hairy 1, } \\
\text { Esr5, Nrarp, } \\
\text { Bowline, Chick } \\
\text { Hairy 1, Mouse } \\
\text { Hes 1, human } \\
\text { HES4 }\end{array}$ & $\begin{array}{l}\text { Mouse Hes } 1 \text { and human HES4 } \\
\text { may be components of } \\
\text { segmentation clock. The other } \\
\text { genes encode factors } \\
\text { downstream of the } \\
\text { segmentation clock. Some of } \\
\text { them are involved in setting the } \\
\text { antero-posterior polarity of } \\
\text { forming somites }\end{array}$ & $\begin{array}{l}\text { In Xenopus, the } 3 \text { 'UTR of Hairy } 2 a \\
\text { confers instability on a reporter mRNA. } \\
\text { The expression pattern of Hairy } 2 a \text { or } \\
\text { Bowline was recapitulated in transgenic } \\
\text { embryos with the appropriate promoter } \\
\text { and a 3'UTR of one of these genes, but } \\
\text { not with a 3'UTR of a stable mRNA. }\end{array}$ & {$[60,61]$} \\
\hline $\begin{array}{l}\text { Xenopus } \mathrm{Su}(\mathrm{H}) \\
\text { (homologue of } \\
\text { mammalian } \\
\text { Rbpj) }\end{array}$ & $\begin{array}{l}\text { Binds to Notch intracellular } \\
\text { domain to stimulate expression } \\
\text { of Notch target genes }\end{array}$ & $\begin{array}{l}\text { mRNA instability is conferred by } \\
\text { association with the RNA-BP Celfl. A } \\
\text { specific impairment of the interaction } \\
\text { between Celfl and } S u(H) \text { mRNA causes } \\
\text { segmentation defects. }\end{array}$ & {$[65,67]$} \\
\hline
\end{tabular}


(a)

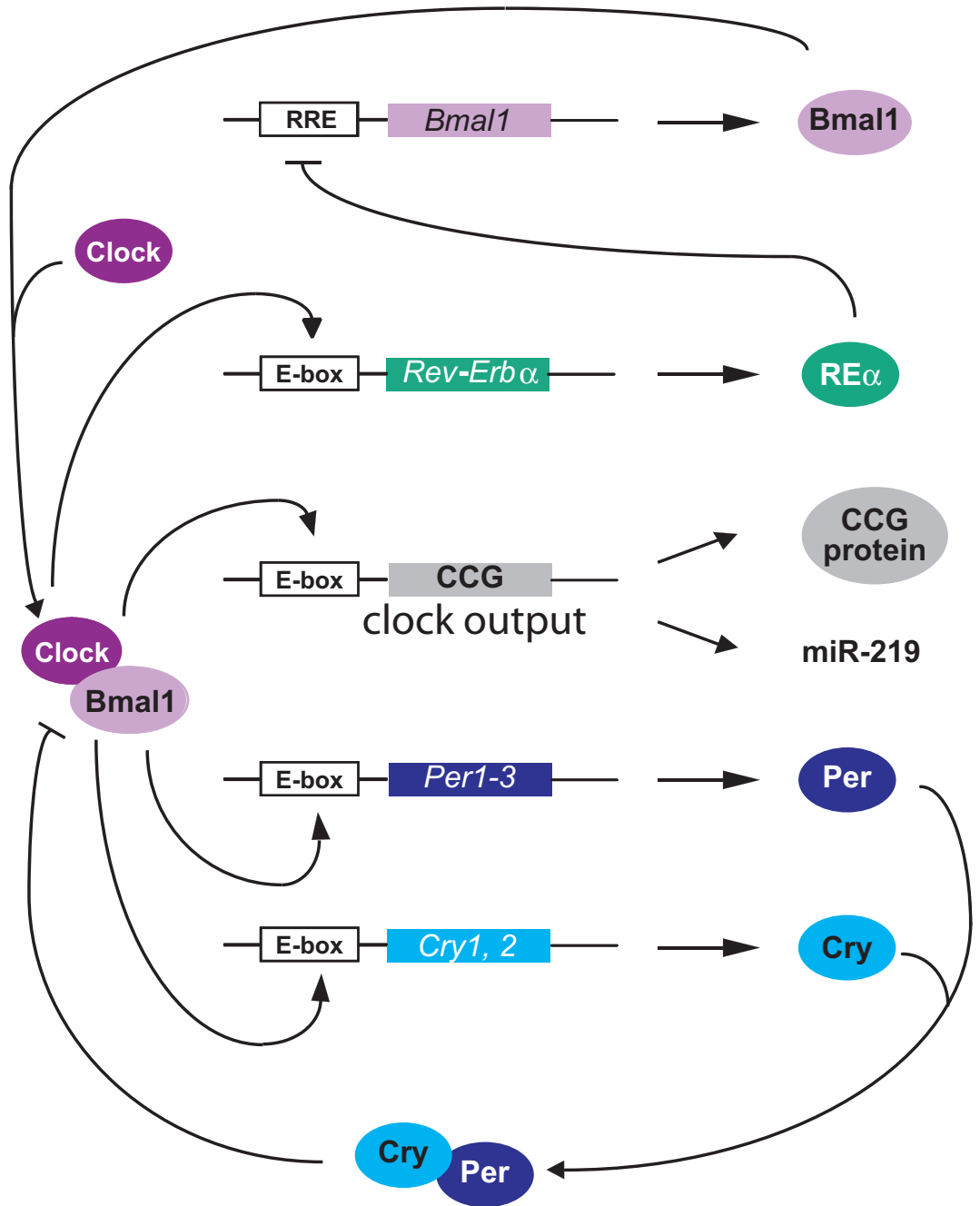

(b)

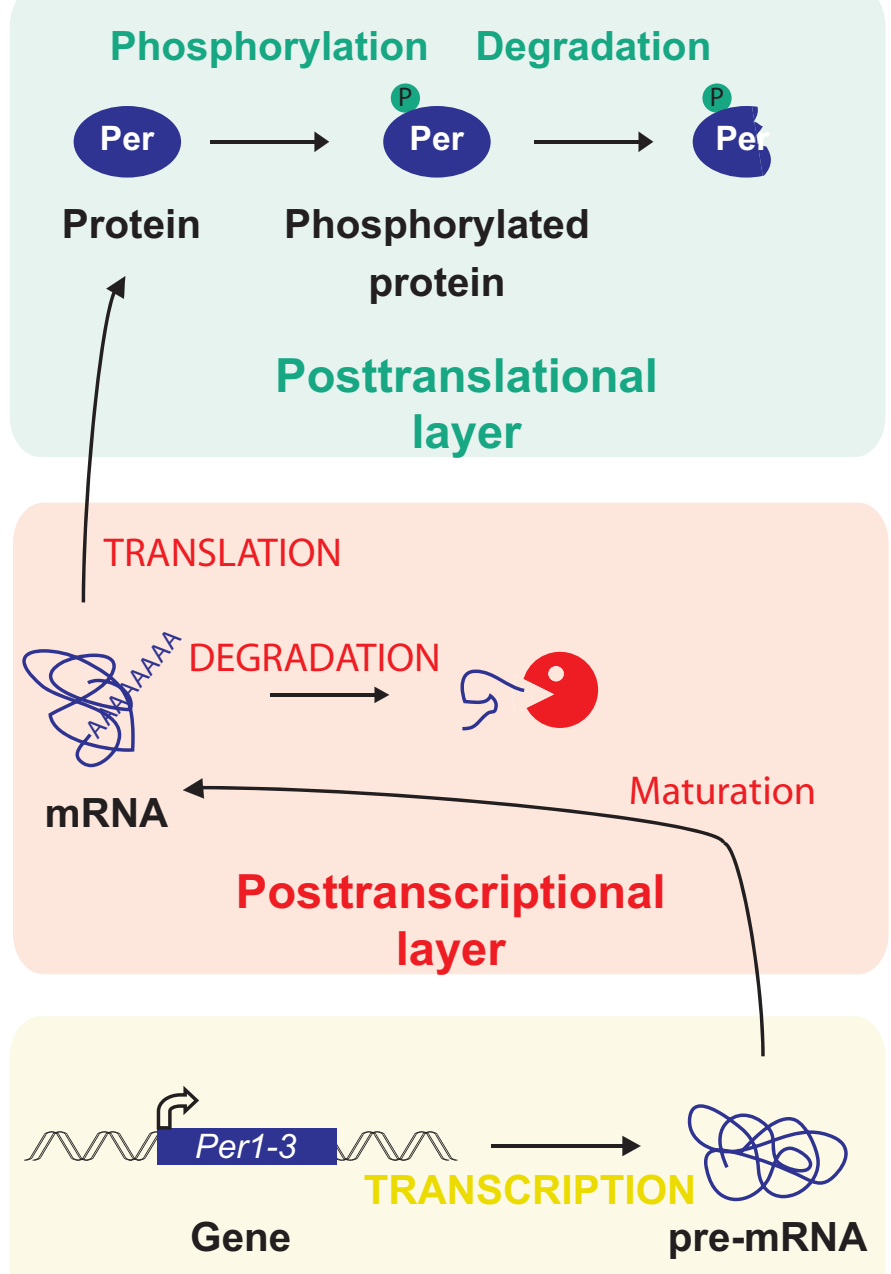

Transcriptional layer

FIGURE 1 
(a)

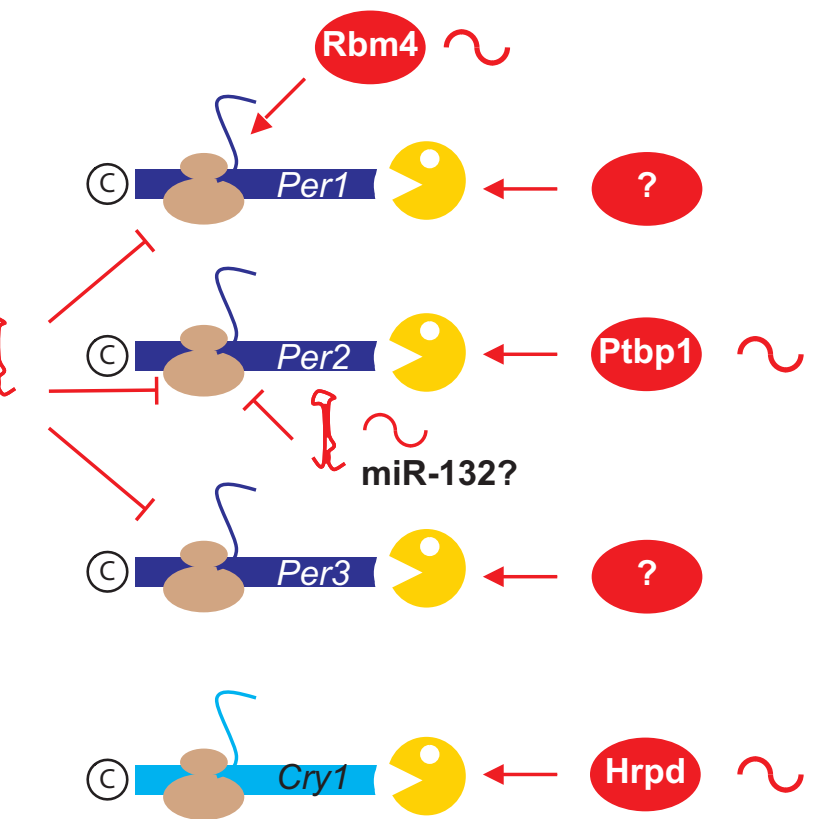

(b) Syncrip $\sim$

FIGURE 2 


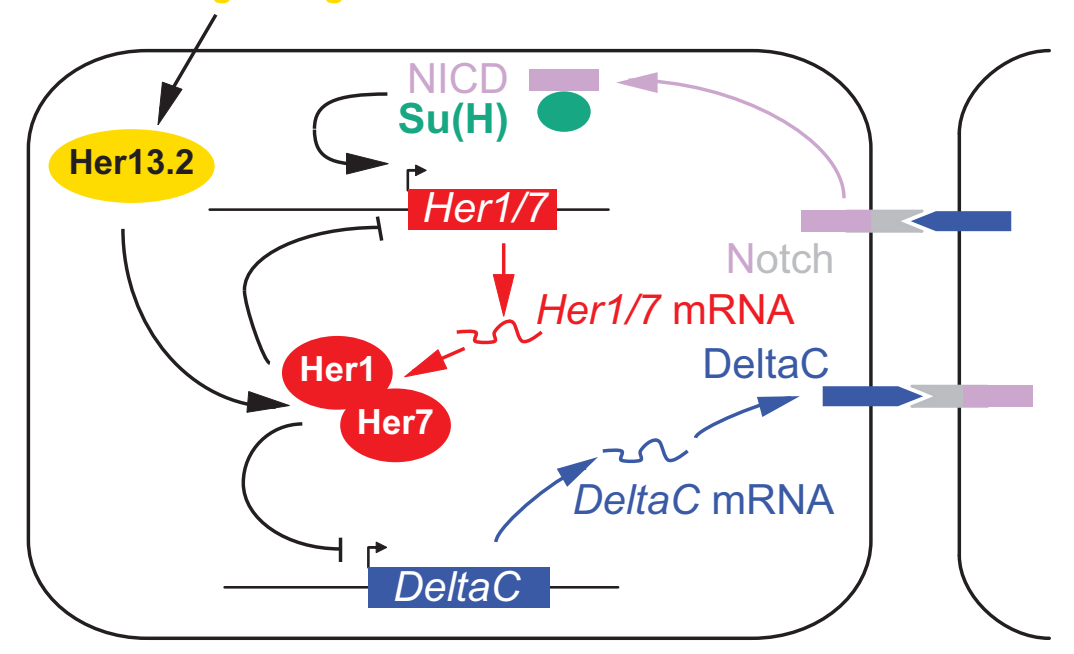

FIGURE OF BOX 2 


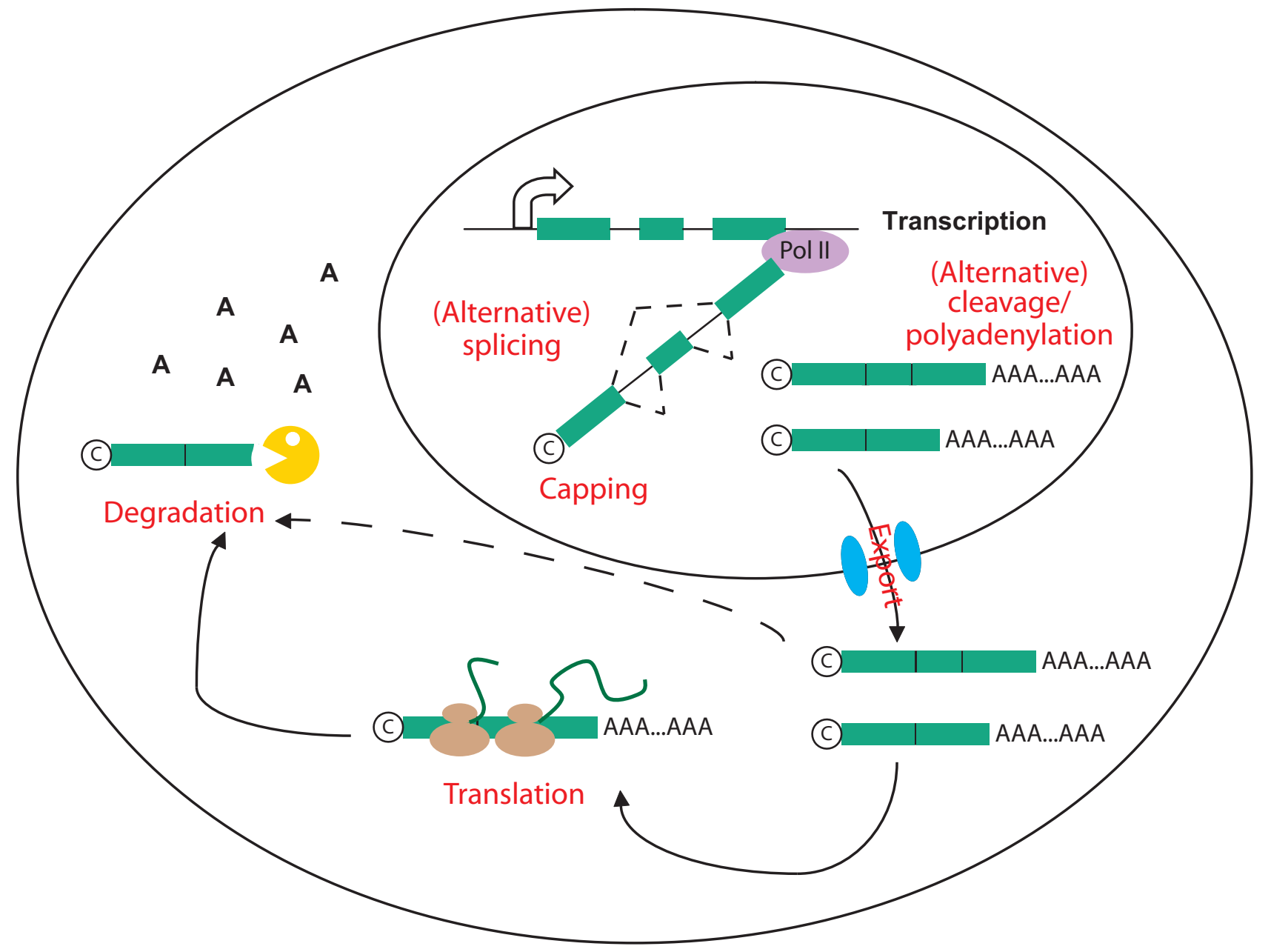

FIGURE OF BOX 3 